\title{
O paradoxo do planejamento das ações para a prevenção do HIV/AIDS em uma escola pública
}

The paradox of the action planning for preventing HIV/AIDS in a public school La paradoja de la planificación de las acciones para la prevención del VIH/SIDA en una
escuela pública

Recebido: $19 / 07 / 2019$

Aprovado: 28/11/2019

Publicado: 17/02/2020
Tiago Sousa Paiva ${ }^{1}$ Marcia Rosa da Costa ${ }^{2}$ Morgana Thais Carollo Fernandes ${ }^{3}$ Camila Neumaier Alves ${ }^{4}$ Luiza Maria de Oliveira Braga Silveira ${ }^{5}$

Este estudo tem como objetivo analisar o planejamento das práticas educativas para a prevenção do HIV/Aids realizadas com estudantes de uma escola pública da região sul do Brasil. Esta é uma pesquisa qualitativa desenvolvida com seis profissionais de uma equipe de Estratégia de Saúde da Família envolvidos com atividades educativas em escola pública, através de análise de conteúdo temático e diário de campo, no ano de 2016. Emergiram duas categorias: Organização do conteúdo e materiais educativos; e, Fluxo do planejamento. Evidenciou-se, que os materiais e conteúdos utilizados não dialogavam com a realidade dos estudantes, pois traziam valores distintos da comunidade e que o fluxo do planejamento era unidirecional. Assim, é necessário que os educadores planejem momentos de educação em saúde que dialoguem com a realidade e o contexto dos educandos e que as ações sejam construídas com a participação da comunidade escolar.

Descritores: Promoção da saúde; Doenças sexualmente transmissíveis; Estudantes.

This study aims to analyze the planning of educational practices for the prevention of HIV/AIDS carried out with students from a public school in southern Brazil. This is a qualitative research conducted with six professionals of a team of the Family Health Strategy involved with educational activities in public school, through thematic content analysis and field diary, in 2016. Two categories emerged: Content Organization and educational materials; and planning flow. It was evident that the materials and content used were not consistent with the students' reality, as they brought different values of the community and that the planning flow was unidirectional. Thus, it is necessary for educators to plan health education moments that dialogue with the reality and context of the students and that actions are built with the participation of the school community.

Descriptors: Health promotion; Sexually transmitted diseases; Students.

Este es un estudio cuantitativo que tiene como objetivo analizar los valores de familia de estudiantes universitarios portugueses y brasileños. Se investigó a 605 universitarios siendo 225 de la Universidad de Madeira (Portugal) y 380 de la Universidad de Fortaleza (Brasil). Se ha utilizado la dimensión familiar del Cuestionario adaptado del European Values Survey, abarcando cuestiones sobre actitudes de los padres, número de hijos y cualidades a enseñar a los niños/jóvenes. La mayoría de los participantes eran mujeres y solteras, presentando a los estudiantes brasileños scores más altos en relación a las actitudes anticuadas de los padres sobre los principios morales y el número de hijos, dando importancia a las cualidades coraje, responsabilidad, respeto, perseverancia, fe, generosidad, mientras que los portugueses otorgan mayor importancia a la economía. Los resultados permiten inferir que las creencias, los valores y las cualidades de los jóvenes reflejan la familia, la sociedad envolvente y el mundo globalizado.

Descriptores: Actitud; Valores sociales; Estudiantes; Educación superior; Familia.

1. Enfermeiro. Especialista em Saúde da Família. Mestre em Ensino na Saúde. Docente no Centro Universitário Ritter dos Reis (UniRitter), Porto Alegre/RS, Brasil. ORCID: 0000-0001-7765-2355 E-mail: sousats@hotmail.com

2. Pedagoga. Mestre e Doutora em Educação. Docente do Programa de Pós Graduação em Ensino na Saúde da Universidade Federal de Ciências da Saúde de Porto Alegre (UFCSPA), Porto Alegre/RS, Brasil. ORCID: 0000-0003-3340-0644 E-mail: lmarciarc@ufcspa.edu.br

3. Enfermeira. Especialista em Saúde Pública. Especialista em Gestão de Redes de Atenção à Saúde. Mestre e Doutora em Pediatria e Saúde da Criança. Docente do UniRitter, Porto Alegre/RS, Brasil. ORCID: 0000-0001-7765-2355 E-mail: morganafernandes@yahoo.com.br

4. Enfermeira. Especialista em Cuidado Pré-Natal. Mestre e Doutora em Enfermagem. Docente no UniRitter, Porto Alegre/RS, Brasil. ORCID: 0000-0002-6455-3689 E-mail: camilaenfer@gmail.com

5. Psicóloga. Mestre e Doutora em Psicologia. Professora Adjunta do departamento de Psicologia e do Programa de Pós Graduação em Ensino na Saúde da UFCSPA, Porto Alegre/RS, Brasil. ORCID: 0000-0002-9531-8251 E-mail: luizabs@ufcspa.edu.br 


\section{INTRODUÇÃO}

$\mathbf{N}$

o início da epidemia do HIV/AIDS, a falta de conhecimento acerca das formas de transmissão da infecção colaborou para a "transmissão" de informações equivocadas que partiam de ações programáticas. Recomendava-se evitar relações sexuais com pessoas que aparentassem estar contaminadas e a diminuição do número de parceiros sexuais; bem como a identificação e caracterização de "grupos de risco", que no imaginário social eram potenciais transmissores do vírus ${ }^{1}$. Com isso, no campo da educação em saúde, historicamente, as práticas de prevenção para o HIV/AIDS foram construídas a partir de um modelo de ensino vertical e que desconsiderava o contexto e as subjetividades que permeavam as relações entre as pessoas.

Os jovens estão no centro das práticas educativas que visam trabalhar a prevenção da AIDS. No intervalo de 2005 até 2014 a taxa da doença entre os jovens de 15 a 19 anos mais que triplicou no país ${ }^{2}$.

Nesse contexto, a escola é um espaço privilegiado para a realização de práticas educativas, por se tratar de um local para o estabelecimento de relações intersubjetivas favoráveis à promoção da saúde e possível de construir respostas para situações desafiadoras para a sociedade $^{3}$. Entretanto, durante encontros de educação em saúde, os estudantes devem ser compreendidos como sujeitos constituídos historicamente em determinado território que por definição contempla a escola, mas, também, o espaço geográfico, histórico, cultural, social e econômico onde se inserem de forma dinâmica ${ }^{4}$.

Pode-se considerar que o aumento das desigualdades sociais, a diminuição de políticas públicas e a baixa disponibilidade de serviços de saúde, também, contribuem para o aumento da vulnerabilidade ao HIV/AIDS, elevando o número de casos entre os jovens ${ }^{5}$. Uma possibilidade de compreender melhor esse fenômeno é através do quadro teórico da vulnerabilidade que surgiu em resposta à discriminação e estigmatização que pessoas infectadas sofriam, a partir da criação do conceito de "grupos de risco".

As diferentes situações de vulnerabilidade dos indivíduos e coletivos podem ser particularizadas numa avaliação articulada das dimensões individual, social e programática ${ }^{6}$. A dimensão individual está relacionada à quantidade e qualidade de informações que as pessoas dispõem sobre os problemas de saúde. A dimensão social diz respeito à disponibilidade de recursos de ordem material, cultural e política que estão disponíveis em determinadas sociedades. Já a dimensão programática, está relacionada à oferta de serviços de saúde, educação, de bem-estar social e cultural. Na dimensão programática, tema central deste estudo, avalia-se o grau e a qualidade dos serviços, programas, políticas públicas e os recursos disponíveis. Inclui-se nesta dimensão o estímulo à participação e à autonomia dos indivíduos para a superação das dificuldades que produzem vulnerabilidades ao HIV7 .

Esta pesquisa filia-se ao referencial da vulnerabilidade e na ideia da educação como prática de liberdade e teve como objetivo analisar o planejamento das práticas educativas para a prevenção do HIV/AIDS realizadas com estudantes de uma escola pública da região sul do Brasil.

\section{MÉTODO}

Trata-se de um estudo descritivo-exploratório de natureza qualitativa que é capaz de incorporar a questão do significado e intencionalidade como inerentes aos atos realizados; e compreender lógicas internas de grupos, instituições, movimentação social e processos históricos ${ }^{8}$.

O estudo foi realizado com profissionais envolvidos com ações de educação em saúde e selecionados a partir dos seguintes critérios: atuar em ações educativas dentro da escola; ter disponibilidade de tempo e aceitar participar da pesquisa. 
O projeto de pesquisa foi apresentado para a Secretaria de Educação, para a Secretaria de Saúde e para os profissionais da equipe da ESF que desenvolvem atividades, por meio do Programa de Saúde na Escola (PSE). Posteriormente, a proposta foi apresentada para a direção da escola, pois, a observação das práticas educativas foi realizada neste contexto, considerando ser uma instituição de ensino situada em uma região do município que apresenta elevada carência de recursos socioeconômicos. Após o aceite dessas instâncias o projeto foi submetido e aprovado por Comitê de Ética com CAAE 43503515.9.0000.5345 e número de parecer 1.074.246.

Uma vez aceito, os profissionais da equipe de saúde assinaram o Termo de Consentimento Livre e Esclarecido (TCLE), obedecendo aos procedimentos para realização de pesquisas com seres humanos (conforme resolução 466/2012 do CONEP/CNS); e a escola assinou um termo de anuência para sua participação.

A coleta de dados ocorreu entre os meses de janeiro e março de 2016 e contou com dois instrumentos: um diário de campo das observações sistemáticas não estruturadas das práticas educativas; e, uma entrevista semiestruturada com vistas a se conhecer: como é planejado o momento de educação em saúde na escola para a prevenção do HIV/AIDS; como se dá a participação dos diferentes profissionais da equipe que se envolvem com essas atividades; de quem partiu a demanda acerca da prevenção do HIV/AIDS; o que os profissionais da equipe pensam sobre a participação da direção e dos professores; que aspectos são levados em consideração para o planejamento das ações e como é realizada a escolha das atividades educativas.

As entrevistas foram gravadas em áudio e posteriormente transcritas para análise do material. As ações educativas foram realizadas com estudantes de uma turma do $5^{\circ}$ ano do ensino fundamental, com a temática da prevenção do HIV/AIDS. A observação foi do tipo não estruturada, partindo da questão inicial: observar as práticas educativas, para a prevenção do HIV/AIDS. A coleta de dados aconteceu.

Os dados foram submetidos à análise de conteúdo ${ }^{9}$, que, como procedimento metodológico é bastante utilizado em estudos qualitativos e análise de materiais deste tipo, apresenta fases distintas que se organizam em torno de três polos cronológicos: a pré - análise; a exploração do material e; o tratamento dos resultados, inferência e interpretação.

\section{RESULTADOS}

Os participantes da pesquisa foram: 01 enfermeiro e 05 agentes comunitárias de saúde. Uma breve caracterização está apresentada no Quadro 1, observando-se que todos, apesar de atuarem há mais de sete anos como profissionais, possuem entre 7 meses e 3 anos de atuação em práticas de prevenção na escola.

Quadro 1. Participantes de ações de planejamento das práticas educativas para a prevenção do HIV/AIDS. Rio Grande do Sul, 2018.

\begin{tabular}{|c|c|c|c|}
\hline Participantes & $\begin{array}{c}\text { Tempo de atuação } \\
\text { profissional }\end{array}$ & $\begin{array}{c}\text { Tempo de atuação nas práticas } \\
\text { educativas na escola }\end{array}$ & Idade \\
\hline ACS 1 & 7 anos & 7 meses & 28 anos \\
\hline ACS 2 & 7 anos & 3 anos & 36 anos \\
\hline ACS 3 & 7 anos & 2 anos & 51 anos \\
\hline ACS 4 & 7 anos & 2 anos & 35 anos \\
\hline ACS 5 & 13 anos & 1 ano & 47 anos \\
\hline Enfermeiro & 9 anos & 2 anos & 32 anos \\
\hline
\end{tabular}

A partir do referencial de análise do material, neste estudo serão apresentados os dados referentes a um eixo temático o qual foi denominado de "Planejamento das Práticas Educativas". Ao serem analisadas as respostas deste eixo temático, emergiram duas categorias: Organização do conteúdo e materiais educativos; e Fluxo do planejamento. 
A categoria "Organização do conteúdo e materiais educativos" agrupou as respostas que se referem aos procedimentos utilizados pelos participantes para a seleção e organização de conteúdos e materiais que são utilizados nas práticas educativas. Segundo uma ACS e o enfermeiro, tal organização se dá a partir de momentos de reunião:

Utilizamos dos nossos conhecimentos teóricos, nossas práticas e montamos um projeto né, com vídeos, com atividades né, tentando incluir eles (alunos) em atividades para poder fazer o trabalho em grupo [...] (ACS 3).

A gente vai muito do nosso dia a dia, do que a gente percebe. Para no futuro ele (o aluno) já ter o conhecimento e não vir a adquirir nenhum vírus e, o pior, desenvolver o HIV/aids (enfermeiro).

Também na análise dos diários de campo, em relação ao conteúdo desta categoria, a observação da realização das práticas educativas possibilitou que se conhecesse o desfecho do seu planejamento dentro da sala de aula. Pode-se exemplificar essa forma de organização dos conteúdos e dos materiais educativos, a partir da cena, da qual as ACS chegam na sala de aula e se inicia a organização do ambiente.

Nesse instante, observou-se quais as ferramentas metodológicas que estavam previstas para serem utilizadas durante a realização das práticas educativas; dentre elas destacou-se: vídeos instrucionais sobre o tema em questão e cartazes que reforçam as informações técnicas e biológicas, acerca do HIV, que, mais tarde seriam reproduzidas no vídeo.

Também, observou-se que uma funcionária da escola auxiliava as ACS na preparação do material audiovisual e na disposição das carteiras, que estavam todas enfileiradas, reproduzindo a organização de uma sala de aula tradicional. Já as ACS prepararam os materiais (cartazes e vídeo) que dariam subsídio para a discussão sobre a prevenção do HIV/Aids.

0 fato de uma funcionária da escola estar preparando o ambiente demonstrou que a equipe tinha feito um contato prévio com a direção da escola para solicitar os recursos que haviam sido preestabelecidos e pactuados durante o planejamento no serviço de saúde. No entanto, até o momento do início da atividade não tinha sido observado indícios da participação dos professores ou da direção da escola, tampouco, o envolvimento dos alunos em relação ao planejamento da atividade.

A segunda categoria chamada "Fluxo do planejamento" emergiu das respostas dos profissionais que revelaram que o planejamento da prática educativa era feito na unidade de saúde pela equipe de ESF e, que somente depois era apresentado para escola, sem muitos espaços para contribuições e transformações, como se exemplifica nas falas dos ACS:

Bom, a gente faz reunião, programa o material, o que a gente vai apresentar para os alunos, apresenta para o nosso coordenador, que é o enfermeiro no caso, aí depois a gente apresenta lá na escola para as professoras, diretoras e a gente apresenta para os alunos (ACS 5);

Quando a gente vai fazer algum trabalho na escola nós reunimos material, cada um traz um material que tem, as ideias que teve [...] traz os assuntos, olhamos todas juntas e, daí vamos fazendo uma seleção de qual que a gente vai usar ou não, daí a gente leva para a escola (ACS 4).

Conforme os dados do diário de campo na observação, constataram-se que a participação da escola se limitava a oferta do espaço físico, disponibilização de horários e dos demais recursos que seriam necessários à prática educativa; e que o envolvimento dos alunos estaria condicionado ao "uso" dos conteúdos e matérias que os profissionais haviam levado para serem trabalhados na sala de aula.

Por sua vez, não se observou a interação dos profissionais da saúde com as professoras que estavam acompanhando a turma, bem como, que o momento educativo e as dinâmicas realizadas com os alunos eram protagonizados pelos profissionais de saúde. Um exemplo disso foi a utilização de um "vídeo educativo" durante o encontro que apresentava informações básicas em relação as formas de contaminação e prevenção do HIV/AIDS.

Nesse momento, explicitou-se o desinteresse dos alunos em relação as informações que estavam sendo reproduzidas através do vídeo, de modo que, os profissionais mais uma vez assumiram o papel de protagonistas e reforçaram as explicações em torno da forma de transmissão e prevenção do HIV/Aids, antes do encerramento da atividade. 


\section{DISCUSSÃO}

Os dados apresentados a respeito da organização do conteúdo e dos materiais educativos sugerem, a partir das falas dos participantes, que existe uma concepção de educação em saúde que tem influenciado o planejamento de suas práticas educativas. Tanto a ACS 3 e o Enfermeiro afirmaram que fazem uso dos conhecimentos produzidos em suas práticas cotidianas, de forma que se tornem subsídios para o planejamento e para o desenvolvimento do encontro com os estudantes, revelando a primazia de seus conhecimentos e de sua experiência.

Em relação a isso, sabe-se que o conhecimento produzido a partir do campo profissional, principalmente, na Estratégia Saúde da Família (ESF) pode fazer emergir questões importantes para o desenvolvimento de uma pedagogia favorável à construção da autonomia do educando e, também, ser capaz de dialogar com a realidade destes indivíduos.

Há necessidade de uma "pedagogia de prevenção e para a cidadania" na prevenção do HIV, de modo que, o modelo de educação libertadora mostra-se a escolha mais oportuna ${ }^{10}$. Isso porque, essa concepção de educação em saúde, atrelada ao conhecimento produzido no cotidiano dos trabalhadores da ESF, pode ser um dos caminhos para que sejam operadas novas formas de prevenção do HIV/Aids no âmbito da Atenção Primaria à Saúde (APS), pela qual, conforme estudo ${ }^{11}$, serão contempladas práticas educativas que considerem as pessoas como sujeitos de direito à saúde integral, a partir das suas singularidades.

Já nos anos 1970 e 1980, Paulo Freire ${ }^{12}$ trouxe à tona a importância de a educação construir-se no contexto do indivíduo para assim produzir significados e transformar o sujeito e, consequentemente, tornar-se libertadora, afirmando ainda que a realidade social é um produto da ação dos homens. A noção de educação freireana parece dialogar com a intenção que os profissionais manifestaram durante as entrevistas acerca do planejamento.

Entretanto, a observação do encontro com os estudantes revelou o contrário. Ou seja, com base nas respostas acerca do planejamento encontrou-se incoerências entre o conteúdo que estava presente na fala dos profissionais e as atitudes e metodologias que foram empregadas durante a realização da prática educativa.

Isso pois, os materiais e o conteúdo que haviam sido reunidos durante o planejamento, foram utilizados sem diálogo com as dúvidas e necessidades que os estudantes poderiam trazer. $\mathrm{Na}$ ocasião, percebeu-se que o método de utilização desses recursos não fomentava a participação dos escolares, tampouco, aproximava-se da realidade dos mesmos. Quando isso ocorre, pode haver uma prática educativa inócua, mostrando-se incapaz de contribuir com o desenvolvimento de atitudes conscientes nas cenas de maior exposição ao HIV.

Incluir a compreensão do referencial da vulnerabilidade no cotidiano das práticas dos profissionais da APS pode contribuir para a utilização dos conhecimentos que estes profissionais possuem da comunidade de onde desenvolvem suas atividades ${ }^{13}$. Na observação da prática educativa, mesmo com a realização de dinâmicas e de questionamentos direcionados à turma percebeu-se que as ACS, como coordenadoras do grupo, não conseguiram ampliar a discussão em torno de assuntos polêmicos e trazidos pelos adolescentes, como as questões relacionadas ao gênero. Além disso, a forma que o cenário educativo foi organizado pode não ter sido favorável ao desenvolvimento de uma prática de educação em saúde emancipatória e problematizadora.

As carteiras na sala de aula reproduzia um cenário tradicional de ensino, que coaduna com a seleção e organização dos materiais para o encontro. Ou seja: com pouco ou nenhum espaço para as contribuições dos estudantes; sem nenhum protagonismo.

Sobre as práticas educativas no campo da saúde, dependendo da concepção de educação em saúde dos trabalhadores, pode-se observar uma lógica disciplinar, hierarquizadora e controladora como a que foi referida por Foucault na obra "Vigiar e Punir". Em relação ao contexto escolar, Foucault problematizou que os mecanismos de poder, também, poderiam ser encontrados em instituições de ensino como as escolas ${ }^{14}$. 
Nesse sentido, esta mesma lógica disciplinar pode estar permeando a relação entre os indivíduos envolvidos no processo de ensino-aprendizagem. Ademais, poderá criar barreiras para o desenvolvimento de atitudes conscientes em relação às situações que vulnerabilizam indivíduos e coletivos, frente a epidemia do HIV/Aids.

A superação de modelos tradicionais de ensino, também, passa pela formação no campo da saúde. Nesse contexto de mudanças, a formação dos profissionais da saúde deveria ser menos centrada na transmissão de informações técnicas e mais direcionada para a compreensão acerca das problemáticas que permeiam a epidemia. A exemplo disso, é preciso valorizar a construção do conhecimento e da reflexão acerca das questões relacionadas aos significados, os sentidos e os aspectos culturais e sociais que permeiam a epidemia do HIV/Aids ${ }^{13}$.

Partindo do processo de territorialização, uma das atribuições da ESF é realizar ações de educação em saúde para a prevenção de agravos e promoção da saúde na área de abrangência de cada equipe, que corresponde, também, ao território da escola ${ }^{4}$. Nesse contexto, as atividades se potencializam quando formuladas a partir do local onde os escolares vivem, pois é deste lugar que as principais problemáticas relacionadas à vulnerabilidade ao HIV/Aids emergem, e de onde o planejamento das práticas educativas poderia ser estruturado.

Com esse entendimento, o planejamento das práticas educativas deve ser desenhado a partir dos saberes que orientam a equipe, mas, sobretudo, com vistas à forma que a equipe se comunica com a comunidade que vive no território. No entanto, no campo das ações que vislumbram o contexto escolar como cenário de prática, nem sempre esses conhecimentos dialogam com as reais necessidades dos estudantes.

Verificou-se também o fato das ações educativas estarem centradas na doença, num modelo clínico. Sugere-se tal ideia pela fala de um dos entrevistados a respeito da contaminação pelo HIV/AIDS. Nesta fala, o enfermeiro refere que, no planejamento da prática educativa, deve prever uma educação para que no futuro os estudantes não vivam com HIV/Aids. Uma pesquisa ${ }^{15}$ destacou que tal analise não se trata de diminuir a importância e a gravidade da infecção, mas de problematizar o fato de que quando coloca-se o vírus e o adoecimento como centro do planejamento das práticas educativas, há risco de operar uma ação que não contribua com a ruptura do modelo clínico hegemônico no campo da prevenção da Aids.

Além disso, pode-se reforçar estigmas e preconceitos que historicamente estão atrelados as pessoas acometidas pela doença. Portanto, faz-se importante pensar o planejamento da prática educativa sem que o HIV/Aids seja o acontecimento mais importante ou o principal evento na vida do indivíduo acometido pela enfermidade. Com isso, avança-se para a compreensão das demais dimensões e problemáticas na vida e no contexto do sujeito. Para além disso, inclui-se as dimensões do referencial da vulnerabilidade, exaltando que elementos de distintas ordens (Individual, social e programáticas) estão presentes nas trajetórias dos sujeitos e de sua condição de saúde geral.

Desde o início da epidemia do HIV/AIDS surgiram uma série de ideias permeadas de preconceito e exclusão, onde a prevenção aparecia como a melhor saída para superar esse problema ${ }^{16}$. E, ainda hoje, as práticas de prevenção são vistas como um dos meios para o enfrentamento dessa problemática ${ }^{17}$. No entanto, para que ocorra o sucesso prático das ações, o modelo de atenção à saúde deve estar em consonância e respeitar a realidade da população do território.

Deste modo, amplia-se a possibilidade de materializar, através das práticas educativas, um fazer que privilegie as subjetividades, o contexto e que, também, supere as ideias que colocam a problemática da infecção do HIV/Aids no centro da vida dos indivíduos. Dito isso, emerge a reflexão de que outros cenários da vida podem ser produtores de vulnerabilidade ao HIV/Aids, como por exemplo os contextos de violência urbana.

Existem outros cenários produtores de vulnerabilidade; porém, quando se trata da violência, um estudo ${ }^{18}$ apontou que pessoas que vivem em territórios de maior vulnerabilidade 
ao HIV/Aids, geralmente, possuem trajetórias de vida com significativas marcas e graus de violência que impactam diretamente na capacidade que elas possuem de lidarem e conseguirem superar situações difíceis ocasionadas pela infecção. Discutir sobre isso é importante, pois, cenários de violência podem ser contextos de opressão e de violação de direitos e, por isso, ampliam a vulnerabilidade ao HIV/Aids.

Considera-se assim importante refletir acerca dos distintos cenários produtores de vulnerabilidade durante o planejamento das práticas educativas, pois, quando o foco do encontro entre educador e educando deixa de ser a doença e passa a ser o contexto e a vida das pessoas, amplia-se a possibilidade do sucesso da ação.

Em relação a categoria "Fluxo do planejamento", evidenciou-se que o encontro entre os trabalhadores da saúde (educadores) e os estudantes (educandos) foi construído na lógica e na direção do campo da saúde para o campo da educação, dando um sentido unidirecional para o planejamento. A problemática em torno do planejamento das práticas educativas ser realizado de forma unidirecional não recai, somente, na questão de a ação ser construída de forma unidisciplinar, mas, também, no fato de essa atividade reverberar no contexto do educando, de forma que desconsidere a cultura e os saberes imbricados na realidade deste indivíduo.

Trata-se da soberania de um saber, de um conteúdo e da supremacia da área da saúde "ensinando" no território da educação. A escola e os professores poderiam também empoderarse deste saber ou colaborar numa ação educativa de prevenção, pois possuem saberes sobre práticas educativas, sobre os alunos, sobre a realidade dos mesmos e da escola.

$\mathrm{Na}$ atualidade, essa hierarquia muitas vezes se reproduz no contexto das práticas dos profissionais da saúde, de modo que, no cenário desta pesquisa os "leigos" em assuntos do campo da saúde são os alunos e os professores da escola. Paulo Freire ${ }^{12}$ chamou esse tipo de prática de ação antidialógica. Ele usa o termo "invasão cultural" e chama a atenção para as situações em que os "invasores" (supostos educadores), que se inserem no contexto cultural dos "invadidos" (os educandos) impõem sua visão de mundo. Enquanto isso, a criatividade do educando vai sendo tolhida. Nesse contexto, "os invasores modelam; os invadidos são modelados. Os invasores optam; os invadidos seguem sua opção".

Ao realizar um planejamento na lógica linear e unidirecional, observa-se uma ação antidialógica, como a exposição do "vídeo educativo" na qual os alunos mostravam sinais de desinteresse em torno do conteúdo que estava sendo apresentado. Tal situação pode estar ancorada no fato de que nem durante ou após a apresentação do vídeo, os profissionais oportunizaram a problematização sobre o tema. Além disso, o desinteresse dos alunos pode ser explicado pelo caráter biomédico do conteúdo, com termos técnicos e desconectados da realidade dos jovens.

O objetivo da educação em saúde, por sua vez, não é o de informar para a saúde, mas transformar saberes existentes. Sendo assim, faz-se importante a reflexão acerca das formas que se constroem as práticas educativas para a prevenção do HIV/Aids no contexto escolar ${ }^{19}$. Nesse sentido, a pedagogia do oprimido pode ser utilizada como ferramenta metodológica para se pensar em uma pedagogia da prevenção, principalmente, no contexto de um país onde uma parte das pessoas, ainda, vive na pobreza ${ }^{10}$. Tal cenário de pauperização, normalmente, está atrelado a situações de violação de direitos humanos que ampliam as diferentes dimensões da vulnerabilidade, como a epidemia do HIV/Aids.

Essa análise propõe uma reflexão a respeito do paradoxo que parece produzir-se entre o discurso dos participantes e as práticas empregadas nas intervenções educativas com os jovens. Acreditou-se que o fato dos profissionais que planejaram a prática educativa serem os mesmos que "vivem na pele" a realidade dos adolescentes foco da intervenção, produziria uma maior aproximação entre o conteúdo, a metodologia empregada, com os alunos e professores da escola.

Porém, mesmo que em determinada medida alguns profissionais tenham manifestado consciência em relação a questões do contexto partilhado, o fato de esse assunto não ser 
pautado durante o encontro com os estudantes sinaliza que essa problemática ainda não se mostra um ponto de reflexão importante a ser associado como uma das vias produtoras de vulnerabilidade ao HIV/Aids. Com isso, o cenário do planejamento das práticas educativas evidenciou a dicotomia que ainda existe entre o saber da equipe e os saberes produzidos no cotidiano da vida dos estudantes e da escola.

\section{CONCLUSÃo}

Durante muito tempo as práticas educativas para a prevenção do HIV/AIDS, no cenário escolar, estiveram ancoradas em concepções de ensino tradicionais e, com isso, mostravam-se cada vez mais ineficazes. No entanto, tal contexto ainda pode ser percebido na atualidade, principalmente, em instituições de ensino como as escolas públicas.

Os resultados desta investigação evidenciaram que as ações desenvolvidas através de iniciativas governamentais como as que emergiram por meio do PSE, para a prevenção do HIV/Aids, são planejadas de forma unidirecional, ou seja, partem do campo da saúde para o campo da educação, e que os conteúdos utilizados durante a ação não dialogam ou colaboram para o desenvolvimento da cidadania e para a formação de consciência, frente a atitudes que aumentam a vulnerabilidade a epidemia do HIV/Aids que vem atingindo, principalmente, os jovens.

O método de ensino utilizado pelos profissionais da saúde com os estudantes da turma baseava-se em modelos tradicionais de educação, na qual, não se oportunizava espaços para a crítica e reflexão. Por conta disso, torna-se necessário pensar sobre essas questões no âmbito da gestão das práticas educativas para prevenção de enfermidades como o HIV/Aids e, também, nos espaços de promoção da saúde, de modo que essas atividades sejam produzidas a partir de uma pedagogia progressista e capaz de colaborar com transformações nos indivíduos e nos contextos onde os mesmos encontram-se inseridos.

Essa investigação limitou-se a análise das ações dos profissionais da saúde no contexto de práticas educativas dentro de uma escola pública. Sendo assim, novos estudos são importantes, como aqueles voltados a conhecer como a escola e os professores percebem/avaliam as ações do PSE na escola.

\section{REFERÊNCIAS}

1. Gianna MC, Kalichman A, Paula I, Vilma C, Shimma E. Políticas públicas e prevenção das DST/Aids: ontem, hoje e amanhã. In: Paiva V, organizador. Vulnerabilidade e direitos humanos - prevenção e promoção da saúde: da doença à cidadania. 1ed. Curitiba: Juruá; 2012.

2. Ministério da Saúde (Brasil). Secretaria de Vigilância em Saúde. Departamento de DST, Aids e Hepatites Virais. HIV AIDS 2018. Boletim Epidemiológico [Internet]. 2018 [citado em 05 mar 2018]; 49(53):1-66. Disponível em: http://www.aids.gov.br/pt-br/pub/2018/boletim-epidemiologicohivaids-2018

3. Souza PL, Pereira CS, Nogueira MLS, Pereira DB, Cunha GM, Moler FO. Projetos PET-Saúde e Educando para a Saúde: construindo saberes e práticas. Rev Bras Educ Med. [Internet]. 2012 [citado em 05 mar 2018]; 36(1 Supl 1):172-7. Disponível em http://www.scielo.br/pdf/rbem/v36n1s1/v36n1s1a24.pdf 4. Ministério da Saúde (Br). Secretaria de Atenção à Saúde. Departamento de Atenção Básica. Saúde na escola [Internet]. Brasília, DF: Ministério da Saúde; 2009 [citado em 5 mar. (Cadernos de Atenção básica; v. 24). Disponível em: http://bvsms.saude.gov.br/bvs/publicacoes/saude_escola_cab24.pdf

5. Marques Junior JS, Gomes R, Nascimento EF. Masculinidade hegemônica, vulnerabilidade e prevenção ao HIV/AIDS. Ciênc Saúde Colet. [Internet]. 2012 [citado em 05 mar 2018]; 17(2);511-2. Disponível em: http://www.scielo.br/pdf/csc/v17n2/a24v17n2.pdf

6. Ayres JRCM, França Júnior I, Calazans GJ, Saletti Filo HC. O conceito de vulnerabilidade e as práticas em saúde: novas perspectivas e desafios. In: Czeresnia D, Freitas CM. Promoção da saúde: conceitos, reflexões e tendências. Rio de Janeiro: FIOCRUZ; 2003. 176p.

7. Ayres JRCM, Calazans GJ, Saletti Filho HC, França-Júnior I. O risco, vulnerabilidade e práticas de prevenção e promoção da saúde. In: Campos GWS, Minayo MCS, Akerman M, Drumond Júnior M, 
Carvalho YM, organizadores. Tratado de saúde coletiva. São Paulo: Hucitec; RJ: Ed Fiocruz; 2006. p. 375417.

8. Minayo MCS. O desafio do conhecimento: pesquisa qualitativa em saúde. 12ed. São Paulo: Hucitec; 2010.

9. Bardin L. Análise de conteúdo. 3ed. Lisboa: Edições 70; 2011. 229 p.

10. Paiva V. Fazendo arte com a camisinha: sexualidades jovens em tempos de AIDS. São Paulo: Summus; 2000.309p.

11. Paiva V, Calazans G, Segurado AAC. Entre indivíduos e comunidades. In: Paiva V, Calazans G, Segurado AAC, coordenadores. Vulnerabilidade e direitos humanos - prevenção e promoção da saúde: da doença à cidadania. 1ed. Curitiba: Juruá; 2012. p. 9-24.

12. Freire P. Pedagogia do oprimido. 8ed. Rio de Janeiro: Paz e Terra; 2013. 218p.

13. Ferraz DAS, Nemes MIB. Prevenção das DST e Aids na Atenção Primária à Saúde: reflexos sobre as práticas a partir do estudo de uma unidade de saúde da família. In: Paiva V, Calazans G, Segurado AAC, coordenadores. Vulnerabilidade e direitos humanos - prevenção e promoção da saúde: da doença à cidadania. 1ed. Curitiba: Juruá; 2012. p. 271-320.

14. Foucault M. Vigiar e punir: nascimento da prisão. Tradução de Raquel Ramalhete. Petrópolis, RJ: Vozes; 1987. 288p

15. Scherer MDA, Marino SRA, Ramos FRS. Rupturas e resoluções no modelo de atenção à saúde: reflexões sobre a Estratégia de Saúde da Família com base nas categorias Kuhnianas. Interface (Botucatu, Online) [Internet]. 2005 [citado em 05 mar 2018]; 9(16):53-66. Disponível em: http://www.scielo.br/pdf/icse/v9n16/v9n16a05.pdf

16. Mendonça LGT. Modelos teóricos como subsídios da prática da promoção da saúde em DST/AIDS no quadro da vulnerabilidade e dos Direitos Humanos. In: Paiva V, Calazans G, Segurado AAC, coordenadores. Vulnerabilidade e direitos humanos - prevenção e promoção da saúde: da doença à cidadania. 1ed. Curitiba: Juruá; 2012. p. 73-100.

17. Ayres JR, Paiva V, Buchalla CM. Direitos humanos e vulnerabilidade na prevenção e promoção da saúde: uma introdução. In: Paiva V, Calazans G, Segurado AAC, coordenadores. Vulnerabilidade e direitos humanos - prevenção e promoção da saúde: da doença à cidadania. 1ed. Curitiba: Juruá; 2012. p. 9-22.

18. Silva JM, Silva CRC. HIV/Aids e violência: da opressão que cala à participação que acolhe e potencializa. Saúd Soc. [Internet]. 2011 [citado em 05 mar 2018]; 20(3):635-46. Disponível em: http://www.scielo.br/pdf/sausoc/v20n3/10.pdf

19. Souza IPM, Jacobina, RR. Educação em saúde e suas versões na história brasileira. Rev Baiana Saúde Pública [Internet]. 2009 [citado em 05 mar 2018]; 33(4):618-27. Disponível em: http://files.bvs.br/upload/S/0100-0233/2009/v33n4/a010.pdf

\section{CONTRIBUIÇÕES}

Luiza Maria de Oliveira Braga Silveira e Tiago Sousa Paiva participaram da concepção do estudo, obtenção dos dados e análise, redação e revisão crítica. Marcia Rosa da Costa, Morgana Thais Carollo Fernandes e Camila Neumaier Alves colaboraram na revisão crítica.

\section{Como citar este artigo (Vancouver)}

Paiva TS, Costa MR, Fernandes MTC, Alves CN, Silveira LMOB. O paradoxo do planejamento das ações para a prevenção do HIV/AIDS em uma escola pública. REFACS [Internet]. 2020 [citado em inserir dia, mês e ano de acesso]; 8(1):18-26. Disponível em: inserir link de acesso. DOI: inserir link do DOI.

\section{Como citar este artigo (ABNT)}

PAIVA, T. S.; COSTA, M. R.; FERNANDES, M. T. C.; ALVES, C. N.; SILVEIRA, L. M. O. B. 0 paradoxo do planejamento das ações para a prevenção do HIV/AIDS em uma escola pública. REFACS, Uberaba, MG, v. 8, n. 1, p. 18-26, 2020. Disponível em: inserir link de acesso. Acesso em: inserir dia, mês e ano de acesso. DOI: inserir link do DOI.

\section{Como citar este artigo (APA)}

Paiva, T.S., Costa, M.R., Fernandes, M.T.C., Alves, C.N. \& Silveira, L.M.O.B. (2020). 0 paradoxo do planejamento das ações para a prevenção do HIV/AIDS em uma escola pública. REFACS, 8(1), 18-26. Recuperado em: inserir dia, mês e ano de acesso de inserir link de acesso. DOI: inserir link do DOI. 